\title{
BeSS, the official Be Star Spectra database
}

\author{
Bertrand de Batz ${ }^{1}$, Coralie Neiner ${ }^{1}$, Michèle Floquet ${ }^{2}$ and \\ François Cochard ${ }^{3}$ \\ ${ }^{1}$ LESIA, Observatoire de Paris-Meudon, France \\ email: B.deBatz@obspm.fr \\ ${ }^{2}$ GEPI, Observatoire de Paris-Meudon, France \\ ${ }^{3}$ Shelyak Instruments, Les Roussets, 38420 Revel, France
}

\begin{abstract}
We present the status of the BeSS database, which contains a catalogue of all known classical Be stars and a large collection of their spectra obtained at any wavelength, any epoch, and from various sources, from amateur astronomer spectra to professional high-resolution high signal-to-noise echelle spectra. Efficient data retrieval in such a heterogeneous data collection is possible with a wide range of selection criteria thanks to their storage in the fits format and via a web interface (http://basebe.obspm.fr) as well as via the Virtual Observatory. BeSS already contains over 49000 spectra and has allowed the detection of several outbursts.
\end{abstract}

Keywords. astronomical data bases: miscellaneous, catalogs, stars: activity, stars: emission-line, Be, stars: variables: other

\section{The BeSS database}

BeSS contains the catalog of all known (2026) Be stars, continuously kept up-to-date, with available fundamental parameters and their references. Anyone willing to share his/her spectra is encouraged to submit those spectra via the dedicated interface. The spectra are carefully checked before becoming public in BeSS. Over 49000 spectra of about 600 Be stars and 63 different sources are already available. The stellar information and spectra stored in BeSS can be used, e.g., for the study of the history of $H_{\alpha}$ emission in an individual Be star, statistical studies of Be star properties or outbursts, the preparation of Be star observations, ...
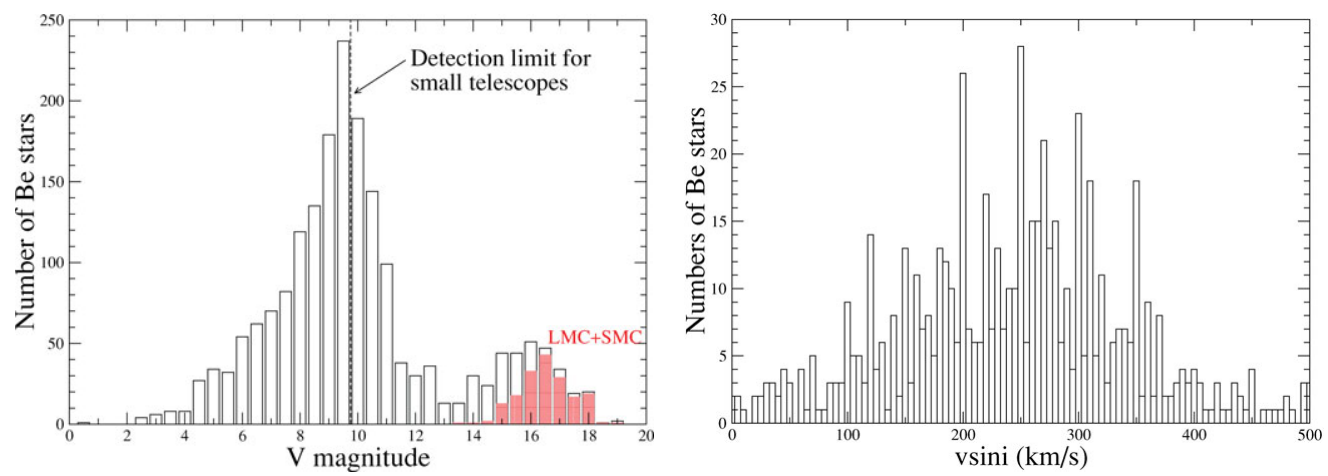

Figure 1. Left panel: distribution in $\mathrm{V}$ magnitude of all Be stars with known $\mathrm{V}$ magnitude. Right panel: distribution of projected rotational velocity for all Be stars with derived vsini. 


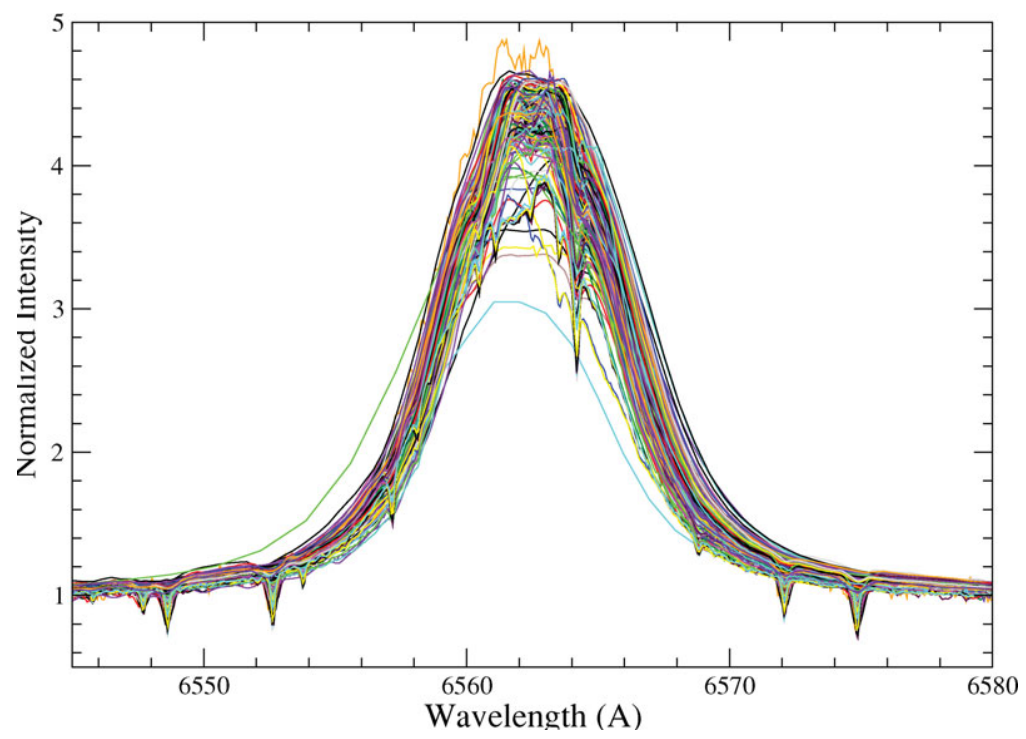

Figure 2. The $187 \mathrm{H}_{\alpha}$ spectra available in BeSS for the star $\gamma$ Cas.

\section{Statistics of Be stars}

Figure 1 shows examples of a statistical use of BeSS. The left histogram shows the distribution in magnitude of all Be stars in the BeSS catalog with known V magnitude. A clear observational bias is seen at the detection limit of small telescopes. Be stars from the LMC and SMC VLT surveys are also visible on the right side of the histogram (in shaded bars). The right histogram shows the distribution of projected rotational velocity (vsini in $\mathrm{km} . \mathrm{s}^{-1}$ ) for all Be stars in the BeSS catalog with derived vsini. The distribution peaks around vsini $=250 \mathrm{~km} \cdot \mathrm{s}^{-1}$.

\section{Study of individual Be stars}

Figure 2 shows an example of all $187 \mathrm{H}_{\alpha}$ spectra available in the BeSS database for the star $\gamma$ Cas. These spectra have been obtained by 20 different observers, including 15 amateur observers, during a 19-year period (1991-2010). More spectra are available in Bess for this star at other wavelengths. Variability in the strong emission at $\mathrm{H}_{\alpha}$ is clearly observed in $\gamma$ Cas and corresponds to the change of structure of the dense circumstellar disk of the Be star. In other Be stars, several outbursts have been detected thanks to BeSS spectra (see Cochard et al., these proceedings).

\section{Conclusions}

The BeSS database is a unique tool for the long-term study of individual Be stars at various wavelength and the detection of outbursts. It also allows the statistical study of Be stars as a population. Finally, its interactive catalog is very useful for the preparation of observations or the selection of adequate targets for a research program. Scientists wishing to contribute to BeSS are welcome to upload their archival spectra into BeSS. 